\title{
Effect of time-dependent piston velocity program on vortex ring formation in a piston/cylinder arrangement
}

\author{
Michael Shusser \\ Faculty of Mechanical Engineering, Technion, Haifa 32000, Israel \\ Moshe Rosenfeld \\ Faculty of Engineering, Tel Aviv University, Tel Aviv 69978, Israel \\ John O. Dabiri and Morteza Gharib \\ Graduate Aeronautical Laboratories and Bioengineering, California Institute of Technology, \\ Pasadena, California 91125
}

(Received 4 December 2005; accepted 22 February 2006; published online 31 March 2006)

\begin{abstract}
An analytical model describing laminar vortex ring formation in a nozzle flow generator (piston/ cylinder arrangement) proposed previously by the authors is extended to time-dependent velocity programs. The predictions of the model are in good agreement with the available numerical data for impulsive, linear, and trapezoidal velocity programs. We also show that properly scaled vortex circulation is another universal quantity, in addition to the dimensionless energy, related to vortex rings and verify this by comparing with available numerical simulations and experimental results.

(C) 2006 American Institute of Physics. [DOI: 10.1063/1.2188918]
\end{abstract}

\section{INTRODUCTION}

Vortex rings are usually generated in the laboratory by the motion of a piston pushing a column of fluid of length $L$ through an orifice or nozzle of diameter $D$. This results in a separation of the boundary layer at the edge of the orifice or nozzle and its subsequent spiral roll-up.

The piston/cylinder arrangement has been extensively used to address the problem of vortex ring formation., ${ }^{1,2}$ Gharib et al., ${ }^{3}$ in their experimental study of vortex ring formation, addressed the question of the largest circulation that a vortex ring can attain by increasing $L / D$ while keeping the average piston velocity fixed.

It turned out that the pinch-off was always observed to occur at a stroke ratio $(L / D)$ of approximately 4 . This universal time scale was called the "formation number." The existence of this limiting time scale for the pinch-off process (formation number) was tested by generating vortex rings with different jet exit diameters, Reynolds numbers, and exit boundary conditions, as well as with various nonimpulsive piston velocity programs.

Krueger and Gharib ${ }^{4}$ measured the dynamic effect of the vortex ring pinch-off process and discovered that the timeaveraged force generated by a starting flow becomes maxi$\mathrm{mal}$ at the onset of vortex ring pinch-off. Krueger et al. ${ }^{5}$ and Dabiri and Gharib ${ }^{6}$ studied the influence of background flow on the vortex ring formation.

Rosenfeld et al., ${ }^{7}$ Zhao et al., ${ }^{8}$ and Mohseni et al. ${ }^{9}$ studied the vortex ring formation numerically. Rosenfeld et $a l^{7}$ showed that the formation number is strongly dependent on the velocity profile and also to a lesser extent depends on the velocity program (the piston velocity as a function of time). The latter fact was also observed by Gharib et al. ${ }^{3}$ In their experiments the formation number ranged from 3.8-4.2 for an impulsive velocity program (constant piston velocity) but could be as large as 4.5 for time-dependent velocity programs.

Mohseni et al. ${ }^{9}$ suggested that the vortex ring pinch-off can be delayed by changing the nozzle diameter during the production of the ring. It was demonstrated experimentally by Dabiri and Gharib ${ }^{10}$ and Allen and Naitoh ${ }^{11}$ that very thick vortex rings can be obtained by temporally decreasing the nozzle exit diameter during fluid ejection.

Theoretical models of vortex ring formation were proposed by Mohseni and Gharib, ${ }^{12}$ Shusser and Gharib, ${ }^{13}$ Linden and Turner, ${ }^{14}$ Mohseni, ${ }^{15}$ and Kaplanski and Rudi. ${ }^{16}$ Shusser and Gharib ${ }^{13}$ proposed a way of modeling vortex ring formation based on the hypothesis that the pinch-off occurs when the translational velocity of the ring equals the jet flow velocity near the vortex ring. The predictions of the model were in good agreement with available experimental and numerical data for the basic case of constant piston velocity.

Studying vortex ring formation and pinch-off can clarify its importance to propulsion and locomotion in biological systems. ${ }^{4,10}$ Since one often encounters temporal variation of nozzle exit diameter or of starting jet velocity in these systems, ${ }^{4,10}$ it is desirable to develop a model of vortex ring formation that is applicable in more general physical situations than the basic case considered previously.

To achieve this goal, such a model must consider a realistic velocity profile and account for the Reynolds number dependence of the formation number. As a first step in this program, we consider the time-dependent piston velocity.

In the present work, we study the vortex ring formation for linear and trapezoidal velocity programs that were investigated in the numerical simulations of Rosenfeld et $\mathrm{al}^{7}{ }^{7} \mathrm{We}$ will also consider vortex ring circulation for general timedependent velocity programs and show that when properly scaled it is another universal quantity related to vortex rings. 
The last result is compatible with Mohseni and Gharib ${ }^{12}$ and Mohseni et al., ${ }^{9}$ who demonstrated that the scaling should be with respect to invariants of motion.

The plan of the paper is therefore as follows. In Sec. II we briefly review several vortex ring formation models. Shusser and Gharib's model ${ }^{13}$ is extended to time-dependent velocity programs in Sec. III. Finally, universality of the vortex ring circulation is discussed in Sec. IV.

\section{VORTEX RING FORMATION MODELS}

Gharib et $\mathrm{al}^{3}$ defined a nondimensional energy of a vortex ring,

$$
\alpha=\frac{E}{\sqrt{\rho I \Gamma^{3}}} .
$$

Here, $E$ is the vortex ring energy, $I$ is the vortex ring impulse, $\Gamma$ is the vortex ring circulation, and $\rho$ is the density of the fluid.

Using the slug-flow approximation ${ }^{1,2}$ for the basic case of a constant piston velocity $U_{P}$ (the impulse velocity program) and a uniform exit velocity profile

$$
\begin{aligned}
& \Gamma=\frac{1}{2} L U_{P}, \\
& I=\frac{1}{4} \pi D^{2} \rho L U_{P}, \\
& E=\frac{1}{8} \pi D^{2} \rho L U_{P}^{2},
\end{aligned}
$$

Gharib et al. ${ }^{3}$ were able to calculate $\alpha$ as a function of the piston stroke $L$. The authors demonstrated that the pinch-off occurs when dimensionless energy provided by the piston decreases beyond a certain limited value $\alpha_{\lim } \approx 0.33$. This value of $\alpha_{\text {lim }}$ corresponded to the formation number $L / D$ $\approx 4$. For higher values of $L / D$, the energy provided by the piston was less than the vortex ring energy. Gharib et al. ${ }^{3}$ concluded that the pinch-off occurs when the apparatus is no longer able to deliver the energy required for steady vortex ring existence.

Aiming to predict the formation number analytically, Mohseni and Gharib ${ }^{12}$ proposed to approximate the ring as a member of Norbury's family of vortex rings. ${ }^{17}$ This family has one parameter-the dimensionless thickness of the ring $\varepsilon$. Each value of $\varepsilon$ between zero and $\sqrt{2}$ corresponds to one particular member of Norbury's family.

Mohseni and Gharib ${ }^{12}$ used Roberts's formula ${ }^{18}$ to calculate the translational velocity of the ring, which was found to be $U_{P} / 2$ in the slug-flow approximation. Using Norbury's family of vortices ${ }^{17}$ and the slug-flow model, they predicted $\alpha_{\text {lim. }}$.

Shusser and Gharib, ${ }^{13}$ trying to clarify the dynamics of the pinch-off, assumed that the pinch-off occurs when the translational velocity of the ring equals the jet flow velocity near the ring. This hypothesis resulted in the following criterion for the pinch-off:

$$
W=\frac{D^{2}}{4 R^{2}} U_{P}
$$

where $W$ is the translational velocity of the ring, $R$ is the ring radius, and $D$ is the diameter of the cylinder.

Adopting the approach of Mohseni and Gharib, ${ }^{12}$ Shusser and Gharib ${ }^{13}$ approximated the ring as a member of Norbury's family of vortex rings ${ }^{17}$ and utilized Fraenkel's second-order formulas ${ }^{19}$ for these vortices:

$$
\begin{aligned}
& W=B(\varepsilon) \sqrt{\frac{\rho \Gamma^{3}}{\pi I}}, \\
& R=b(\varepsilon) \sqrt{\frac{2 I}{\rho \pi \Gamma}},
\end{aligned}
$$

where

$$
\begin{aligned}
& B(\varepsilon)=\frac{1}{4} \sqrt{1+\frac{3}{4} \varepsilon^{2}}\left[\ln \frac{8}{\varepsilon}-\frac{1}{4}+\frac{3 \varepsilon^{2}}{8}\left(\frac{5}{4}-\ln \frac{8}{\varepsilon}\right)\right], \\
& b(\varepsilon)=\frac{1}{\sqrt{2\left(1+\frac{3}{4} \varepsilon^{2}\right)}} .
\end{aligned}
$$

Shusser and Gharib ${ }^{13}$ obtained a nonlinear equation for the dimensionless energy of the ring at pinch-off as

$$
\alpha=\frac{2 B R^{2}}{D^{2} \sqrt{\pi}}
$$

They showed that the experimental value of $\alpha_{\text {lim }}$ obtained by Gharib et al. ${ }^{3}$ satisfies (10) with the reasonable accuracy of about $6 \%$. The authors concluded that both their approach and that of Gharib et al. ${ }^{3}$ and Mohseni and Gharib ${ }^{12}$ are equivalent.

Shusser et al. $^{20}$ (see also Dabiri and Gharib ${ }^{21}$ ) investigated the influence of a boundary layer on the cylinder wall on vortex ring formation. They solved Eq. (10) using numerical values for properties of Norbury's vortices given in Ref. 17. Though this solution could not be calculated with a high accuracy due to Norbury's data only being available for discrete values of $\varepsilon$, its approximate value was found to be about 0.4 . The authors also verified the accuracy of Fraenkel's approximation ${ }^{19}$ for Norbury's vortices by calculating the formation number for the basic case of the uniform velocity profile and constant piston velocity using both Norbury's data ${ }^{17}$ and Fraenkel's second-order formulas. ${ }^{19}$ The accuracy of Fraenkel's approximation turned out to be very good, the error being less than $2 \%$.

We now proceed to extend the model proposed by Shusser and Gharib ${ }^{13}$ to time-dependent velocity programs. Since the model is based on the slug-flow approximation, the predictions of the model will be compared with numerical results of Rosenfeld et al. ${ }^{7}$ obtained for the uniform velocity profile. 


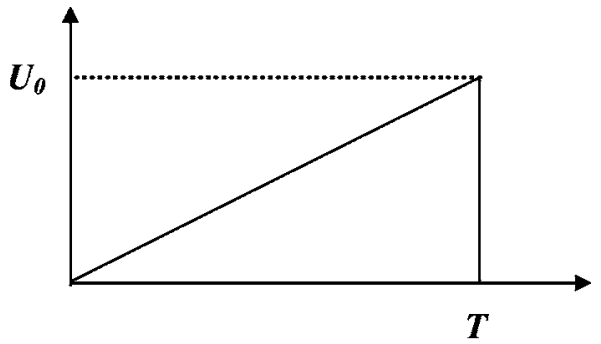

(a)

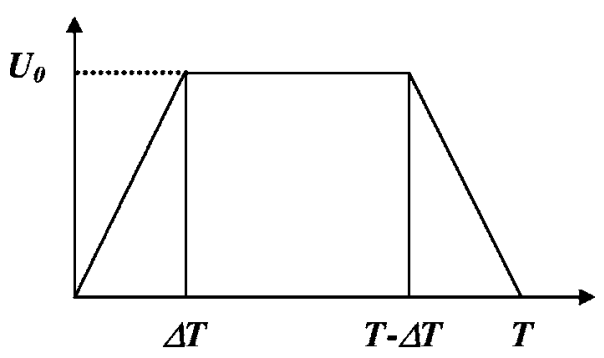

(b)

FIG. 1. Nonimpulse velocity programs: (a) linear; (b) trapezoidal.

\section{TIME-DEPENDENT PISTON VELOCITY}

Consider formation of a laminar vortex ring in a piston/ cylinder arrangement. Following Shusser and Gharib, ${ }^{13}$ we aim to estimate the dimensionless pinch-off time by calculating the translational velocity of the ring. We adopt the slugflow approximation and assume a uniform exit velocity profile. We also approximate the ring as a member of Norbury's family of vortex rings ${ }^{17}$ and utilize Fraenkel's second-order formulas ${ }^{19}$ for these vortices (6)-(9).

Following Shusser et al. ${ }^{20}$ we shall assume for the dimensionless thickness of the ring $\varepsilon=0.4$. This choice of $\varepsilon$ corresponds to $B=0.6987$ and $b=0.6682$. Comparing these values with Norbury's numerical solution, one can see that the accuracy of Fraenkel's approximation is within 1.4\% in this case.

\section{A. Linear velocity program}

Consider a linear program for the piston velocity [see Fig. 1(a)],

$$
U_{P}=U_{0} \frac{t}{T}
$$

The slug-flow approximation for this case is as follows:

$$
\begin{aligned}
& \Gamma=\frac{1}{3} L U_{0}, \\
& I=\frac{1}{6} \pi D^{2} \rho L U_{0} .
\end{aligned}
$$

Calculating the properties of the ring from (6)-(9), one obtains for the radius $R$ and the velocity $W$

$$
R=b D,
$$

$$
W=\frac{B \sqrt{2}}{3 \pi} \frac{U_{P} L}{D} .
$$

Applying the criterion (5), we obtain that the formation number is

$$
\frac{L}{D}=\frac{3 \pi \sqrt{2}}{8 b^{2} B} \approx 5.34 .
$$

The value of the formation number obtained numerically by Rosenfeld et al. ${ }^{7}$ is 5.22 . Therefore, the model provides a good accuracy of $2.3 \%$ for the linear velocity program.

\section{B. Trapezoidal velocity program}

Following Rosenfeld et al., ${ }^{7}$ a symmetric trapezoidal velocity program as shown in Fig. 1(b) was also considered. As in their paper, the final piston stroke ratio was selected to be

$$
\int_{0}^{T} U_{P}(t) d t=6 D
$$

Then, the velocity program is defined by a single parameter

$$
n=\frac{\Delta T}{T} \text {. }
$$

The case $n=0$ corresponds to the impulse program. Obviously, it must be $n \leqslant 0.5$. The condition (17) can be written as

$$
\frac{U_{0} T}{D}=\frac{6}{1-n} .
$$

Repeating the calculation of Sec. III A, one can see that (19) ensures that the first part of the velocity program $(0 \leqslant t \leqslant \Delta T)$ is not long enough for the pinch-off to happen during this time. For the middle part $(\Delta T \leqslant t \leqslant T-\Delta T)$

$$
\begin{aligned}
& U_{P}=U_{0}, \\
& L=U_{0}\left(t-\frac{\Delta T}{2}\right), \\
& \Gamma=\frac{1}{2} U_{0}^{2}\left(t-\frac{2 \Delta T}{3}\right), \\
& I=\frac{\pi D^{2}}{4} \rho U_{0}^{2}\left(t-\frac{2 \Delta T}{3}\right) .
\end{aligned}
$$

Using (5)-(9), we obtain that the dimensional time of the pinch-off $t^{*}$ is

$$
t^{*}=\frac{2 \Delta T}{3}+\frac{\pi \sqrt{2} T(1-n)}{24 b^{2} B} .
$$

This value of time lies within the range $\Delta T \leqslant t^{*} \leqslant T-\Delta T$ if $n \leqslant n_{0}$, where

$$
n_{0}=\frac{24 b^{2} B-\pi \sqrt{2}}{40 b^{2} B-\pi \sqrt{2}} \approx 0.38 .
$$

Substituting (24) into (21), we obtain that for $n \leqslant n_{0}$ the formation number is 


$$
\frac{L}{D}=\frac{n}{1-n}+\frac{\pi \sqrt{2}}{4 b^{2} B} .
$$

The case $n=0$ corresponds to the impulse velocity program. Then,

$$
\frac{L}{D}=\frac{\pi \sqrt{2}}{4 b^{2} B} \approx 3.56 .
$$

For the uniform velocity profile and the impulse velocity program Rosenfeld et al. ${ }^{7}$ obtained the formation number $L / D=3.60$. One sees that our prediction is very close to their numerical results.

If $n>n_{0}$ then the last part of the velocity program $T-\Delta T \leqslant t \leqslant T$ must be analyzed. Then,

$$
\begin{aligned}
& U_{P}=\frac{U_{0}(T-t)}{\Delta T}, \\
& L=U_{0}\left[T-\Delta T-\frac{(T-t)^{2}}{2 \Delta T}\right], \\
& \Gamma=\frac{1}{2} U_{0}^{2}\left[T-\frac{4 \Delta T}{3}-\frac{(T-t)^{3}}{3 \Delta T^{2}}\right], \\
& I=\frac{\pi D^{2}}{4} \rho U_{0}^{2}\left[T-\frac{4 \Delta T}{3}-\frac{(T-t)^{3}}{3 \Delta T^{2}}\right] .
\end{aligned}
$$

Defining

$$
x=\frac{T-t}{\Delta T},
$$

one finds, after some manipulation, that the pinch-off time corresponds to the root of the cubic equation

$$
x^{3}+\frac{\pi \sqrt{2}(1-n)}{8 b^{2} B n} x+4-\frac{3}{n}=0 .
$$

Considering the derivative of the left-hand side of (33), one can show that for $n_{0} \leqslant n \leqslant 0.5$ Eq. (33) has only one real root $x^{*}$ and that this root satisfies $0<x^{*} \leqslant 1$. Then, using (29) and (32), we find the formation number

$$
\frac{L}{D}=6-\frac{3 x_{*}^{2} n}{1-n} .
$$

The relationships (26) and (34) as well as the numerical results of Rosenfeld et al. ${ }^{7}$ are plotted in Fig. 2. For convenience, the linear program case, which can be considered as $n=1$, was also added to this figure though the range 0.5 $<n<1$ does not correspond to any physical situation. We see that the agreement between the theoretical and the numerical results is good (the discrepancy does not exceed $3.8 \%$ ) and one can conclude that despite its simplicity the model gives good predictions for a broad range of different velocity programs.

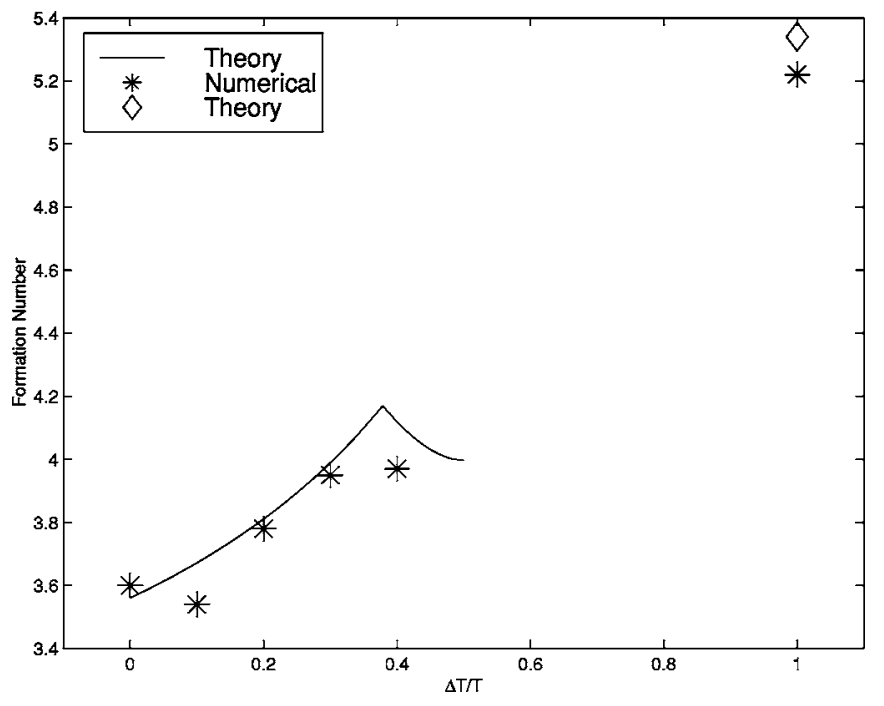

FIG. 2. Formation number for impulse, linear, and trapezoidal velocity programs.

\section{VORTEX RING CIRCULATION}

Rosenfeld et al. (Ref. 7, p. 313), analyzing their numerical simulations for various time-dependent velocity programs, observed that the vortex ring circulation is relatively insensitive to the formation conditions, once its asymptotic state has been reached. The authors conjectured that properly scaled vortex circulation is yet another universal quantity related to vortex rings. We now show that this conjecture is indeed right (see also Mohseni and Gharib ${ }^{12}$ ).

Consider a general time-dependent velocity program,

$$
U_{P}=U_{0} f(t) \text {. }
$$

Using the slug-flow approximation, one obtains for the vortex circulation $\Gamma$ and its impulse $I$

$$
\begin{aligned}
& \Gamma=\frac{1}{2} U_{0}^{2} F(t), \\
& \frac{I}{\rho}=\frac{\pi D^{2}}{4} U_{0}^{2} F(t),
\end{aligned}
$$

where $f(t)$ and $F(t)$ are related by

$$
F(t)=\int_{0}^{t} f^{2}(\tau) d \tau
$$

Using (6) and (7), one can write the pinch-off criterion (5) as

$$
\sqrt{\frac{\Gamma I}{\rho}}=\frac{\pi \sqrt{\pi}}{8 b^{2} B} D^{2} U_{P}
$$

Substituting (36) and (37) in (39) yields

$$
\frac{F(t)}{f(t)}=\frac{\pi}{2 \sqrt{2} b^{2} B} \frac{D}{U_{0}} .
$$

Following Mohseni and Gharib, ${ }^{12}$ we define a dimensionless vortex ring circulation $\Gamma_{\mathrm{nd}}$, where "nd" stands for nondimensional, as follows: 


$$
\Gamma_{\mathrm{nd}}=\Gamma \sqrt[3]{\frac{\rho}{I U_{P}^{2}}} .
$$

Substituting (36) and (37) and using (40), one obtains

$$
\Gamma_{\mathrm{nd}}=\sqrt[3]{\frac{\pi}{16 b^{4} B^{2}}} \approx 1.26 .
$$

Thus, the dimensionless vortex ring circulation defined by (41) is indeed a universal constant for all time-dependent velocity programs. On the other hand, its value will be different for nonuniform exit velocity profiles.

Before comparing the theoretical result (42) with numerical computations, we would like to note that Mohseni and Gharib ${ }^{12}$ also suggested a definition of the dimensionless vortex ring circulation based on the translational velocity of the ring,

$$
\Gamma_{\mathrm{nd}}^{\prime}=\Gamma \sqrt[3]{\frac{\rho}{I W^{2}}} .
$$

Consequently, they showed that only two nondimensional parameters, namely $E_{\text {nd }}$ and $\Gamma_{\text {nd }}$ (or $\Gamma_{\text {nd }}^{\prime}$ ), govern the pinchoff process. Mohseni et al. ${ }^{9}$ verified this numerically.

Using (6), (36), and (37), we obtain that

$$
\Gamma_{\mathrm{nd}}^{\prime}=\sqrt[3]{\frac{\pi}{B^{2}}} \approx 1.86 .
$$

Mohseni and Gharib (Ref. 12, p. 2438) report that the experimental value of $\Gamma_{\text {nd }}^{\prime}$, which they calculated from the set of experiments of Gharib et al., ${ }^{3}$ is 1.75 . On the other hand, Allen and Naitoh ${ }^{11}$ obtained in their experiments values of $\Gamma_{\text {nd }}^{\prime}$ between 1.7 and 2.5 but with a relatively large experimental error of \pm 0.25 . One can conclude that the model predictions are in agreement with experimental results and use numerical calculations for further verification.

To verify the universality of the dimensionless circulation (41), we have compared our results with the data from numerical simulations of Rosenfeld et $a{ }^{7}{ }^{7}$ for the uniform exit velocity profile and impulse, linear, and trapezoidal velocity programs, as given in their Table 1. Though the authors did not report the value of vortex ring impulse, its value can be estimated if one assumes that the ring impulse is equal to the total momentum flux from the exit of the cylinder (Ref. 3, p. 135). Then, the impulse of the ring can be calculated from the total circulation produced by the piston $\Gamma_{T}$ (which is included in Table 1 of Rosenfeld et al. $^{7}$ ) by using the slug-flow approximation,

$$
\frac{I}{\rho}=\frac{\pi D^{2}}{2} \Gamma_{T} .
$$

Normalizing the ring and the total circulations as in Rosenfeld et $a l^{7}$ and using (45), one obtains

$$
\Gamma_{\mathrm{nd}}=\sqrt[3]{\frac{2}{\pi}} \frac{\Gamma}{U_{0} D} \frac{1}{\sqrt[3]{\frac{\Gamma_{T}}{U_{0} D}}}\left(\frac{U_{P}}{U_{0}}\right)^{-2 / 3} .
$$

The ratio of piston to maximum velocity $U_{P} / U_{0}$ in (46) should be calculated at the pinch-off time using the values of

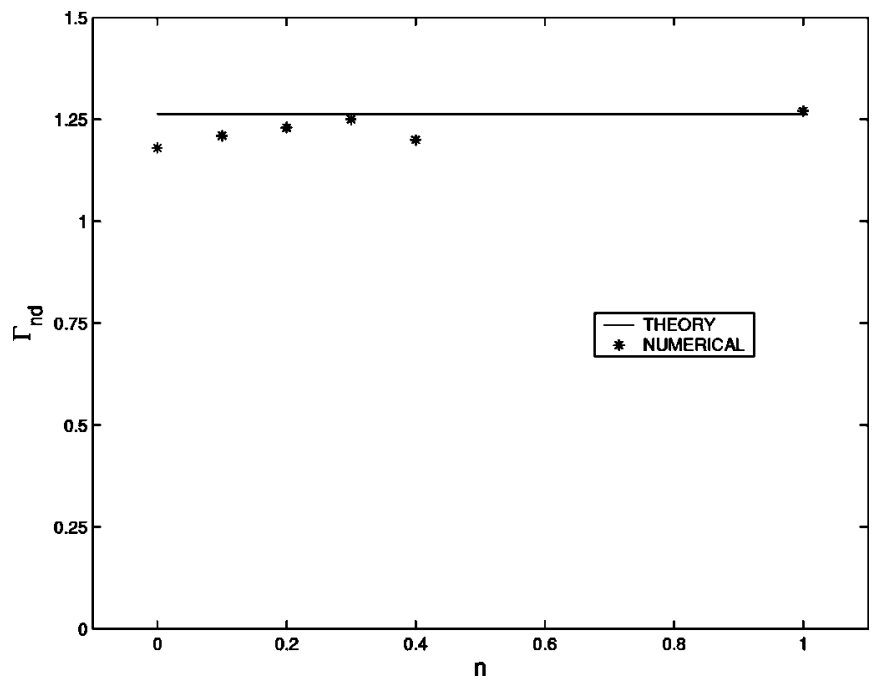

FIG. 3. Comparison of theoretical and numerical results for vortex ring circulation.

the formation number given in Table 1 of Rosenfeld et al. ${ }^{7}$ The result is as follows: for the impulse and trapezoidal velocity programs

$$
\frac{U_{P}}{U_{0}}=1,
$$

for the linear program

$$
\frac{U_{P}}{U_{0}}=\sqrt{\frac{L / D}{6}} .
$$

In (47b), $L / D$ denotes the formation number.

For trapezoidal programs, (47a) means that the pinch-off occurs in the middle part of the program. It should be noted that our theory predicts this for $n \leqslant 0.38$ [see (25)]. That means that for $n=0.4$ the pinch-off must take place very close to the end of the middle part. This is indeed the case, as can be seen from the values of the formation number reported by Rosenfeld et al. ${ }^{7}$ For $n=0.4$, the end of the middle part of the velocity program corresponds to $L / D=4$, while Rosenfeld et al. ${ }^{7}$ obtained in their calculations $L / D=3.97$.

Having computed the value of $\Gamma_{\text {nd }}$ from Rosenfeld et al.'s data, ${ }^{7}$ we plot it in Fig. 3 together with the theoretical value of $\Gamma_{\mathrm{nd}}(42)$. One sees that, despite some variation in $\Gamma_{\text {nd }}$, its value always remains close to the theoretical prediction. The error in the model does not exceed $6.3 \%$ and remains within $4 \%$ for most of the cases. One can conclude that dimensionless circulation of the ring (41) is indeed a universal quantity.

\section{CONCLUSIONS}

We have shown that Shusser and Gharib's model ${ }^{13}$ of vortex ring formation in a piston/cylinder arrangement can be extended to time-dependent velocity programs. It gives good predictions for both linear and trapezoidal velocity programs. 
It follows from the theory that properly scaled vortex ring circulation is yet another universal quantity related to vortex rings. This result is confirmed by available numerical simulations of Rosenfeld et al. ${ }^{7}$

It is interesting to compare the predictions of the theory with the experiments on vortex ring formation with temporal variation of the nozzle exit diameter. ${ }^{10,11}$ According to the theory, increasing the piston velocity increases the formation number and vice versa. Therefore, one would expect higher values of the formation number for the decreasing nozzle exit diameter and lower values of the formation number for the increasing nozzle exit diameter, the static case being somewhere in the middle. Considering, for example, the experiments of Dabiri and Gharib, ${ }^{10}$ we see that this prediction is mostly but not completely confirmed. Dabiri and Gharib ${ }^{10}$ (see their Fig. 11) obtained the highest value of the formation number for their "faster-closing" case, and the values of the formation number for the closing nozzle were considerably higher than those for the opening nozzle. On the other hand, increasing the nozzle exit diameter somewhat increased the formation number with respect to the static case. The reason for this discrepancy is the strong dependence of the formation number on the exit velocity profile. ${ }^{7}$ Temporal variation of nozzle exit diameter changes the exit velocity profile which has an additional effect on the formation number, besides the one caused by acceleration or deceleration of the flow.

\footnotetext{
${ }^{1}$ K. Shariff and A. Leonard, "Vortex rings," Annu. Rev. Fluid Mech. 24, 235 (1992).

${ }^{2}$ T. T. Lim and T. B. Nickels, "Vortex rings." in Fluid Vortices, edited by S. I. Green (Kluwer, Dordrecht, The Netherlands, 1995).

${ }^{3}$ M. Gharib, E. Rambod, and K. Shariff, "A universal time scale for vortex ring formation," J. Fluid Mech. 360, 121 (1998).

${ }^{4}$ P. S. Krueger and M. Gharib, "The significance of vortex ring formation to
}

the impulse and thrust of a starting jet," Phys. Fluids 15, 1271 (2003).

${ }^{5}$ P. S. Krueger, J. O. Dabiri, and M. Gharib, "Vortex ring pinchoff in the presence of simultaneously initiated uniform background co-flow," Phys. Fluids 15, L49 (2003).

${ }^{6}$ J. O. Dabiri and M. Gharib, "Delay of vortex ring pinchoff by an imposed bulk counterflow," Phys. Fluids 16, L28 (2004)

${ }^{7}$ M. Rosenfeld, E. Rambod, and M. Gharib, "Circulation and formation number of laminar vortex rings," J. Fluid Mech. 376, 297 (1998).

${ }^{8}$ W. Zhao, S. H. Frankel, and L. G. Mongeau, "Effects of trailing jet instability on vortex ring formation," Phys. Fluids 12, 589 (2000).

${ }^{9}$ K. Mohseni, H. Ran, and T. Colonius, "Numerical experiments on vortex ring formation," J. Fluid Mech. 430, 267 (2001).

${ }^{10}$ J. O. Dabiri and M. Gharib, "Starting flow through nozzles with temporally variable exit diameter," J. Fluid Mech. 538, 111 (2005).

${ }^{11} \mathrm{~J}$. J. Allen and T. Naitoh, "Experimental study of the production of vortex rings using a variable diameter orifice," Phys. Fluids 17, 061701 (2005).

${ }^{12} \mathrm{~K}$. Mohseni and M. Gharib, "A model for universal time scale of vortex ring formation," Phys. Fluids 10, 2436 (1998).

${ }^{13}$ M. Shusser and M. Gharib, "Energy and velocity of a forming vortex ring," Phys. Fluids 12, 618 (2000).

${ }^{14}$ P. F. Linden and J. S. Turner, "The formation of 'optimal' vortex rings, and the efficiency of propulsion devices," J. Fluid Mech. 427, 61 (2001).

${ }^{15} \mathrm{~K}$. Mohseni, "Statistical equilibrium theory for axisymmetric flows: Kelvin's variational principle and an explanation for the vortex ring pinchoff process," Phys. Fluids 13, 1924 (2001).

${ }^{16}$ F. B. Kaplanski and Y. A. Rudi, "A model for the formation of 'optimal' vortex rings taking into account viscosity,” Phys. Fluids 17, 087101 (2005).

${ }^{17}$ J. Norbury, "A family of steady vortex rings," J. Fluid Mech. 57, 417 (1973).

${ }^{18}$ P. H. Roberts, "A Hamiltonian theory for weakly interacting vortices," Mathematika 19, 169 (1972).

${ }^{19}$ L. E. Fraenkel, "Examples of steady vortex rings of small cross section in an ideal fluid," J. Fluid Mech. 51, 119 (1972).

${ }^{20}$ M. Shusser, M. Rosenfeld, K. Mohseni, and M. Gharib, "On the effect of pipe boundary layer growth on the formation of a laminar vortex ring generated by a piston/cylinder arrangement," Theor. Comput. Fluid Dyn. 15, 303 (2001).

${ }^{21}$ J. O. Dabiri and M. Gharib, "A revised slug model boundary layer correction for starting jet vorticity flux," Theor. Comput. Fluid Dyn. 17, 293 (2004). 